\title{
Calculation of the P- and T-odd spin-rotational Hamiltonian of the PbF molecule
}

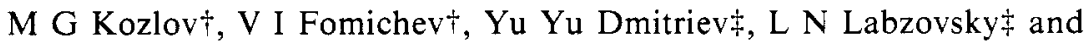 \\ A V Titovt \\ $\dagger$ Leningrad Institute of Nuclear Physics, Academy of Sciences, Leningrad, USSR \\ †eningrad State University, Leningrad, USSR
}

Received 5 January 1987

\begin{abstract}
The electron wavefunctions for two states of the PbF molecule are calculated. For the ground state of the molecule, tensor components of the hyperfine interaction of the valence electron with the nuclear spin of $\mathrm{Pb}$, electric and magnetic dipole moments and electron matrix elements of P-odd and P, T-odd interactions are calculated. In accordance with the calculation an effective spin-rotational Hamiltonian for the molecule is given. The Hamiltonian includes the parity-non-conserving terms and the interaction with the external fields. The spin-rotational spectrum of the PbF molecule is found to be rather complicated; this is connected with the large anisotropy of the hyperfine $A$ tensor. The results of the present work can be used in planning future experiments on the search for $\mathrm{P}$-odd and $\mathrm{P}, \mathrm{T}$-odd phenomena in molecules.
\end{abstract}

\section{Introduction}

It is known that the simultaneous violation of parity $P$ and time reversal invariance $T$ can be larger by several orders of magnitudes for diatomic molecules than for atoms (Sandars 1967). For molecules the enhancement is of the order of $10^{4}-10^{5}$, and it is due to the narrow spacing of energy levels with the opposite parity. This fact stimulated the experiment on the TIF molecule (Wilkening et al 1984) which gave the upper bounds on the electric dipole moment (EDM) of the proton and on some constants of the P, T-odd electron-nuclear interaction (Coveney and Sandars 1983, Sushkov et al 1984).

In experiments of this kind the frequency shift of the magnetic resonance in parallel and antiparallel electronic and magnetic fields is measured. A recent experiment on the $\mathrm{Xe}$ atom (Vold et al 1984) showed that the advantage of the diatomic molecules is partially illusive. This is mainly because of the slow relaxation of the nuclear magnetisation in diamagnetic atoms such as $\mathrm{Xe}$ and $\mathrm{Hg}$. One can measure the frequency shift in atoms with much higher accuracy than in molecules. The gain in accuracy of the frequency shift measurement in atoms is comparable with the enhancement of the effect in diatomic molecules.

Another situation occurs in the case of molecules with non-closed electronic shells. The difference is that the experiments with diamagnetic atoms and molecules give rather weak bounds on the constants of the P, T-odd interactions depending on the electron spin (Flambaum and Khriplovich 1985a). These are the electron-nuclear neutral current interactions, the electron EDM and the interaction of electrons with the 
nuclear magnetic quadrupole moment (Khriplovich 1981). In diatomic molecules with unpaired electrons the interactions mentioned above are enhanced by approximately four orders of magnitude in comparison with atoms (Sushkov and Flambaum 1978, Gorshkov et al 1979, Sushkov et al 1984). The accuracy of the frequency shift measurements differs little for paramagnetic atoms and molecules. Hence, the measurement of the EDM of diatomic radicals seems to be rather promising. What is more, here the anapole moment of the nucleus can be measured (Flambaum and Khriplovich 1985b, Kozlov 1985).

As all $\mathrm{P}$ - and $\mathrm{P}, \mathrm{T}$-odd interactions show a strong $Z$ dependence, it is sensible to choose heavy atoms as the diatomic radicals. Among the possible candidates BiS, $\mathrm{PbX}, \mathrm{HgX}, \mathrm{DyX}$ (here and below $\mathrm{X}$ is a halogen) are usually mentioned. The hyperfine constants of these molecules are of the same order of magnitude or even larger than the rotational constants. It then follows that the spin-rotational spectra of the radicals are complicated, and for an analysis of the $\mathrm{P}$ - and $\mathrm{P}$, T-odd effects a detailed knowledge of the spin-rotational Hamiltonian is needed.

For some diatomic molecules $(\mathrm{HgF}, \mathrm{BaF})$ the parameters of the spin-rotational Hamiltonian in the ${ }^{2} \Sigma_{1 / 2}$ electronic ground state are known from experiment (see references in Kozlov 1985). For heavy molecules such as BiS or $\mathrm{PbX}$ with a ${ }^{2} \Pi_{1 / 2}$ ground state the hyperfine constants have not been measured experimentally and the spin-rotational Hamiltonian is unknown.

In the present article we attempt to estimate the hyperfine constants and electronic matrix elements of the parity violating interactions in the PbF molecule. A semirelativistic method proposed by Dmitriev et al (1985) is used. The method includes a oneparameter fitting of the $\omega$ doubling in two lower states of the molecule. We describe here the details of the calculation of two electronic wavefunctions $|\Pi\rangle$ and $|\Sigma\rangle$. The spin-orbit interaction of the unpaired electron is taken into account semiempirically. Finally, the one-electron orbitals of the wavefunctions are used to calculate the matrix elements of the hyperfine and parity violating interactions.

\section{Calculation of the low-lying electronic states of the PbF molecule}

Relativistic quantum mechanics gives a general basis for an adequate description of the electronic states of heavy molecules such as PbF. Unfortunately at present there are no effective algorithms for molecular calculations in a fully relativistic manner. The difficulties are both technical and theoretical (Kutzelnigg 1984). However, assuming that chemical bonds are mainly formed by the non-relativistic valence electrons, we can consider the inner shells of the relativistic electrons as a frozen core. Then it is natural to introduce an effective core potential (ECP) in the non-relativistic selfconsistent field equations for the valence orbitals. The ECP has to bring the relativistic effects for the valence electrons from the atomic calculations into the molecular calculations. The historical background as well as many theoretical approaches for the ECP method are documented in the literature (Kahn 1984).

To approximate the ECP the numerical four-component orbitals of lead $\varphi_{n, k}$ ( $n$ is the principal quantum number and $k$ denotes other quantum numbers of the relativistic orbitals) were obtained by the Hartree-Fock-Dirac method (computer program by Bratcev et al 1977).

The $6 \mathrm{~s}_{1 / 2}, 6 \mathrm{p}_{1 / 2}, 6 \mathrm{p}_{3 / 2}$ and $5 \mathrm{~d}_{3 / 2}, 5 \mathrm{~d}_{5 / 2}$ orbitals were chosen as valence ones $\left(\varphi_{n, k}^{v}\right)$. The valence orbitals were then substituted by the nodeless pseudo-orbitals $\chi_{k}^{v}$. 
According to the traditional definition of the pseudo-orbitals they were taken as a linear combination of the valence $\varphi_{n, k}^{v}$ and core $\varphi_{n, k}^{c}$ orbitals with the same quantum number $k$ :

$$
\chi_{k}^{\mathrm{v}}=\sum_{n^{\prime}<n} b_{n, n^{\prime}, k}^{\mathrm{c}} \varphi_{n^{\prime}, k}^{\mathrm{c}}+b_{n, k}^{\mathrm{v}} \varphi_{n, k}^{\mathrm{v}} .
$$

The coefficients $b_{n, n^{\prime}, k}^{\mathrm{c}}$ were chosen so as to minimise the functional (Liberman et al 1965):

$$
\int_{0}^{\infty}\left(r^{-\gamma} \frac{\mathrm{d}}{\mathrm{d} r} F_{k}(r)\right)^{2} \mathrm{~d} r
$$

were $F_{k}$ is a large component of the bispinor $\chi_{k}^{v}$ and $\gamma=\left(k^{2}-\alpha^{2} Z^{2}\right)^{1 / 2}$.

The pseudo-orbitals $\chi_{k}^{v}$ do not oscillate at the nucleus and correspond to oneelectron orbitals in the field of the effective charge $Z^{*}=Z-N_{\mathrm{c}}$, where $N_{\mathrm{c}}$ is the number of core electrons.

Since $Z^{*} \ll Z$, the small components of the bispinor $\chi_{k}^{v}$ can be neglected while the remaining nodeless components satisfy the Schrödinger equation with non-singular potentials:

$$
U_{k}=\frac{1}{F_{k}}\left(\varepsilon_{k}+\frac{1}{2} \frac{\mathrm{d}^{2}}{\mathrm{~d} r^{2}}-\frac{1}{2} \frac{k(k+1)}{r^{2}}-\hat{W}_{k}\right) F_{k}
$$

where $\varepsilon_{k}$ is an orbital energy and $\hat{W}_{k}$ is a sum of the Coulomb and exchange operators.

The ECP can be written in the form (Wadt and Hay 1985)

$$
\hat{V}_{\mathrm{ef}}=\sum_{l=0}^{l_{\text {max }}}\left(V_{l}(r)-V(r)\right) \sum_{m}|l, m\rangle\langle l, m|+V(r) .
$$

Here $l_{\max }$ is a maximum angular momentum of the occupied atomic orbitals and $V_{l}$ is obtained by averaging the potentials (3) with $k_{1}=l$ and $k_{2}=-l-1$ :

$$
V_{l}=\left(n_{1} U_{k_{1}}+n_{2} U_{k_{2}}\right) /\left(n_{1}+n_{2}\right)
$$

In this formula $n_{1}$ and $n_{2}$ are the occupation numbers of $F_{k_{1} ; k_{2}}$.

The potential $V(r)$ is usually taken as equal to $V_{l_{\max }+1}$. In our case $l_{\max }=3$ and $V(r)$ is the potential for the $\mathrm{g}$ orbitals.

The ECP (4) was then used in the non-relativistic SCF calculations. The pseudoorbitals $\chi_{k}^{v}$ and the potentials $V_{l}$ were approximated by linear combinations of Gaussian functions. These functions together with the Gaussian functions of fluorine (Danning and Hay 1977) formed the LCAO basis set for the PbF molecule. The SCF calculations were performed with the computer programs MOLECULE (Almlöf 1972) and ALCHEMY (Bagus 1975) slightly modified for ECP calculations (Dmitriev et al 1985).

\begin{tabular}{|c|c|c|c|}
\hline & \multirow[b]{2}{*}{ Experiment } & \multicolumn{2}{|r|}{ Theory } \\
\hline & & This work & Balasubramanian (1985) \\
\hline$D_{0}^{0}$ & 0.1323 & 0.1610 & 0.1628 \\
\hline IP & 0.2756 & 0.2490 & - \\
\hline$E(\Sigma)-E(\Pi)$ & 0.1028 & 0.0803 & 0.0868 \\
\hline
\end{tabular}

Results of these calculations are collected in tables 1 and 2. The dissociation energy and ionisation potential for the ground state and the transition energy between $\Pi$ and

Table 1. Theoretical and experimental values for the dissociation energies, transition energies and ionisation potentials (in au) of $\mathrm{PbF}$. 
Table 2. Coefficients of the MO LCAO expansion for the PbF molecule.

\begin{tabular}{lllllr}
\hline & $\mathrm{s}$ & $\mathrm{p}$ & $\mathrm{d}$ & $\mathrm{s}^{\prime}$ & \multicolumn{1}{c}{$\mathrm{p}^{\prime}$} \\
\hline$|\pi\rangle$ & - & 0.9943 & - & - & -0.2557 \\
$|\sigma\rangle$ & 0.2768 & 0.9955 & 0.03 & 0.3519 & 0.5122 \\
\hline
\end{tabular}

$\Sigma$ states refer to the equilibrium internuclear distance $R=3.888$ au. These results are in agreement with the experimental data (Huber and Herzberg 1979) and the more sophisticated theoretical investigation of $\mathrm{PbF}$ (Balasubramanian 1985).

The molecular orbitals were also expanded in the atomic basis. Coefficients of the expansion for the molecular orbitals of the unpaired electrons in the $\Pi$ and $\Sigma$ states are given in table 2 .

According to the previous consideration, the nodeless pseudo-orbitals $\chi_{k}^{v}$ represent in the molecular calculations real relativistic orbitals $6 \mathrm{~s}_{1 / 2}, 6 \mathrm{p}_{1 / 2}, 6 \mathrm{p}_{3 / 2}$ and $5 \mathrm{~d}_{3 / 2}, 5 \mathrm{~d}_{5 / 2}$ of lead. Hence the molecular orbital of the unpaired electron in $\Pi$ and $\Sigma$ states can be written as

$$
\begin{aligned}
& |\pi, \omega\rangle=\mathrm{p}_{\pi} 3^{-1 / 2}\left(2 \omega 2^{1 / 2}\left|6 \mathrm{p}_{1 / 2}\right\rangle+\left|6 \mathrm{p}_{3 / 2}\right\rangle\right)+\mathrm{p}_{\pi}^{\prime}|2 \mathrm{p}\rangle \\
& |\sigma, \omega\rangle=\mathrm{s}_{\sigma}\left|6 \mathrm{~s}_{1 / 2}\right\rangle+\mathrm{p}_{\sigma} 3^{-1 / 2}\left(-2 \omega\left|6 \mathrm{p}_{1 / 2}\right\rangle+2^{1 / 2}\left|6 \mathrm{p}_{3 / 2}\right\rangle\right)+\mathrm{s}_{\sigma}^{\prime}|2 \mathrm{~s}\rangle+\mathrm{p}_{\sigma}^{\prime}|2 \mathrm{p}\rangle
\end{aligned}
$$

where $\omega$ is the projection of the total angular momentum of the electron on the molecular axis. Coefficients at the fluorine orbitals in (5) and (6) are indicated by the prime and d functions of lead are omitted.

\section{Relativistic corrections to the wavefunction}

The orbitals (5) and (6) correspond to the non-relativistic approximation in which the projection $\Lambda$ of the electron orbital angular momentum $\boldsymbol{L}$ on the molecular axis is a constant of motion. The spin-orbit interaction mixes the states with different values of $\Lambda$ and the same projection $\omega$ of the total electron angular momentum $J^{\mathrm{e}}$. We assume then that the two lowest electronic states correspond to the following orbitals of the unpaired electron:

$$
\begin{aligned}
& |0, \omega\rangle=\xi|\pi, \omega\rangle+\eta|\sigma, \omega\rangle \\
& |1, \omega\rangle=-\eta|\pi, \omega\rangle+\xi|\sigma, \omega\rangle
\end{aligned}
$$

where $\omega= \pm \frac{1}{2}$.

The mixing coefficients may be estimated as

$$
\eta \simeq\left(\varepsilon_{\pi}-\varepsilon_{\sigma}\right)^{-1}\left\langle\sigma, \omega\left|H_{\text {so }}\right| \pi, \omega\right\rangle
$$

where $H_{\text {so }}$ is the spin-orbit interaction. It is rather obvious that the matrix elements of the spin-orbit interaction are mainly determined by the $6 \mathrm{p}$ functions of lead. Hence, the following estimate for the mixing coefficient $\eta$ can be used (Sobelman 1963)

$$
\eta \simeq \frac{\alpha^{2} Z^{2} \mathrm{p}_{\pi} \mathrm{p}_{\sigma}}{6 \nu^{2}\left(\varepsilon_{\pi}-\varepsilon_{\sigma}\right)}=0.25
$$

where $\nu$ is an effective principal quantum number of the $6 \mathrm{p}$ functions. 
One can also estimate the mixing coefficients $\eta$ and $\xi$ by fitting to the experimental values of the $\omega$ doubling (Dmitriev et al 1985). The energy difference between the levels of the $\omega$ doublet is determined by the following matrix element of the total electronic angular momentum $\boldsymbol{J}^{\mathrm{c}}$ :

$$
\Delta_{n} \equiv(-1)^{J+1 / 2} \frac{E_{n, J,-}-E_{n, J,+}}{\left(J+\frac{1}{2}\right)}=2 B\left\langle n, \omega\left|J_{+}^{\mathrm{e}}\right| n,-\omega\right\rangle .
$$

In this formula $E_{n, J, p}$ is the energy of the $\omega$ doublet level, $p$ denotes the parity and $B$ is the rotational constant. Reducing the matrix element of $\boldsymbol{J}^{\mathrm{e}}$ to the matrix element of $\boldsymbol{L}$ and taking the experimental values of $\Delta_{n}$ (Huber and Herzberg 1979) we obtain the following equations for $\eta$ and $\xi$ :

$$
\begin{aligned}
& \eta^{2}+2 \xi \eta L=\Delta_{0} / 2 B=-0.30 \\
& \xi^{2}-2 \xi \eta L=\Delta_{1} / 2 B=1.49 \\
& \xi^{2}+\eta^{2}=1
\end{aligned}
$$

where

$$
L \equiv\left\langle\pi\left|L_{+}\right| \sigma\right\rangle
$$

In principle this system of equations is not compatible. Indeed, it follows from the first two equations (10) that

$$
\xi^{2}+\eta^{2}=1.19
$$

which is not consistent with the third equation (10). This inconsistency determines the quality of the two-level approximation used here. The numerical value for the matrix elements (11) is

$$
L=1.2 \text {. }
$$

Using this value for $L$ we obtain from the equations (10)

$$
\begin{aligned}
& \eta=-(0.19 \pm 0.06) \\
& \xi=0.98 \mp 0.01 .
\end{aligned}
$$

The upper sign in (13) corresponds to the first and third equations of system (10), and the lower sign corresponds to the second and third equations.

Equations (7) and (13) lead to the following expansion for the molecular orbital of the unpaired electron in the ground state

$$
|0, \omega\rangle=a\left|6 \mathrm{~s}_{1 / 2}, \omega\right\rangle+2 \omega b\left|6 \mathrm{p}_{1 / 2}, \omega\right\rangle+c\left|6 \mathrm{p}_{3 / 2}, \omega\right\rangle
$$

where

$$
\begin{aligned}
& a=\eta \mathrm{s}_{\sigma}=-(0.052 \pm 0.016) \\
& b=3^{-1 / 2}\left(2^{1 / 2} \xi \mathrm{p}_{\pi}-\eta \mathrm{p}_{\sigma}\right)=0.90 \mp 0.02 \\
& c=3^{-1 / 2}\left(\xi \mathrm{p}_{\pi}+2^{1 / 2} \nu \mathrm{p}_{\sigma}\right)=0.41 \pm 0.05
\end{aligned}
$$

In this expansion we have omitted the $\mathrm{d}$ functions of lead and the functions of fluorine because they are not used in the following calculations. 


\section{Spin-rotational Hamiltonian of the $\mathrm{PbF}$ molecule}

The parity conserving part of the spin-rotational Hamiltonian for the ground state of $\mathrm{PbF}$ includes the rotational term, electron rotation (Coriolis) and hyperfine interactions

$$
H_{\mathrm{sr}}=B \boldsymbol{J}^{2}-2 B \boldsymbol{J}^{\mathrm{e}} \boldsymbol{J}+\boldsymbol{s}^{\prime} \hat{\boldsymbol{A}} \boldsymbol{I} \text {. }
$$

In this expression $B$ is the rotational constant, $\boldsymbol{J}^{\mathrm{e}}$ and $\boldsymbol{J}$ are electronic and total angular momenta of the molecule, $I$ is the nuclear spin of $\mathrm{Pb}$ and $s^{\prime}$ is the operator of the effective spin in the degenerate subspace $|0, \pm \omega\rangle$. The hyperfine interaction of $s^{\prime}$ with the fluorine spin is small and it is neglected in the present consideration.

The hyperfine interaction tensor $\hat{\boldsymbol{A}}$ is diagonal in the molecular frame of reference: $A_{z z} \equiv A_{\|}, A_{x x}=A_{y y} \equiv A_{\perp}$. As was shown earlier (Kozlov 1985)

$$
\begin{aligned}
& A_{\|}=a^{2} h_{s}+b^{2} h_{1}+c^{2} h_{3}+\left(\frac{8}{3}\right)^{1 / 2} b c h_{1,3} \\
& A_{\perp}=a^{2} h_{s}-b^{2} h_{1}+2 c^{2} h_{3}
\end{aligned}
$$

where $a, b$ and $c$ are determined in (15),

$$
\begin{aligned}
& h_{i}=(-1)^{j+1 / 2-l} \frac{2 j+1}{j(j+1)} \frac{\mu_{\mathrm{N}}}{I} \int_{0}^{\infty} \frac{f_{i} g_{i}}{r^{2}} \mathrm{~d} r \\
& h_{1,3}=2 \times 3^{-1 / 2} \frac{\mu_{\mathrm{N}}}{I} \int_{0}^{\infty} \frac{f_{1} g_{3}+f_{3} g_{1}}{r^{2}} \mathrm{~d} r
\end{aligned}
$$

where $f_{i}$ and $g_{i}$ are the components of the Dirac bispinor

$$
\frac{1}{r}\left(\begin{array}{c}
f_{i} \Omega_{j, l} \\
i g_{i} \Omega_{j, l}
\end{array}\right)
$$

and indices s, 1 and 3 correspond to $6 s_{1 / 2}, 6 p_{1 / 2}$ and $6 p_{3 / 2}$ functions.

Stable isotopes of lead have nuclear spin 0 or $\frac{1}{2}$. In the first case the last term of (16) vanishes and the energies and eigenfunctions of the Hamiltonian $H_{\mathrm{sr}}$ are written as (Sushkov and Flambaum 1978, Dmitriev et al 1985)

$$
E_{J, p}=B J(J+1)+\frac{\Delta}{2}(-1)^{J+1 / 2} p\left(J+\frac{1}{2}\right)
$$

where $\Delta$ is determined by the formula (9) with $n=0$,

$$
|J, M, p\rangle=2^{-1 / 2}\left(|0, \omega\rangle|J, M, \omega\rangle+(-1)^{J+1 / 2} p|0,-\omega\rangle|J, M,-\omega\rangle\right) .
$$

$|J, M, \omega\rangle$ is the rotational wavefunction of a symmetric top.

If $I=\frac{1}{2}$, the total angular momentum of the molecule is $\boldsymbol{F}=\boldsymbol{J}+\boldsymbol{I}$. In this case the spin-rotational energies are:

$$
\begin{aligned}
E_{F, p, \pm}=B(F+ & \left.\frac{1}{2}\right)^{2}+\frac{1}{4} \chi\left(A_{\perp}-\Delta\right) \pm\left(F+\frac{1}{2}\right)^{-1} \\
& \times\left\{\left[\left(B-\frac{1}{2} \Delta \chi\right)\left(F+\frac{1}{2}\right)^{2}-\frac{1}{8}\left(A_{\|}-\chi A_{\perp}\right)\right]^{2}+\frac{1}{16} F(F+1)\left(A_{\|}-\chi A_{\perp}\right)^{2}\right\}^{1 / 2}
\end{aligned}
$$

where

$$
\chi=(-1)^{F} p \text {. }
$$

For the levels with $F=0$ the upper sign has to be taken.

In the experiments on parity non-conservation the lowest levels of the Hamiltonian (16) have to be used. These levels are shown in figure 1.

An interaction of the molecule with external fields is described by the operator

$$
H_{\text {ef }}=\boldsymbol{D E}+\boldsymbol{M} \mathscr{H} \text {. }
$$




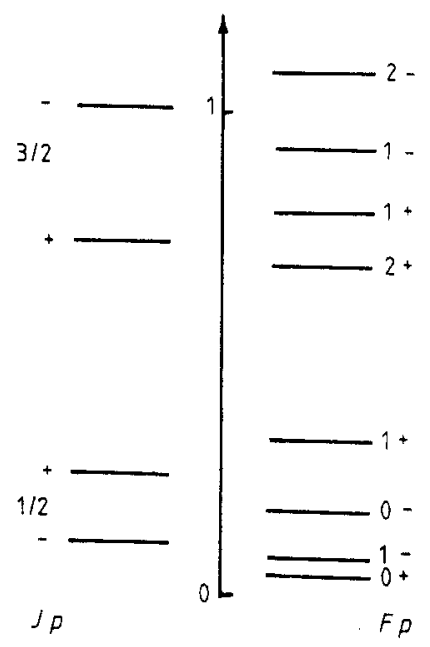

Figure 1. The lower spin-rotational levels (in $\mathrm{cm}^{-1}$ ) for the spin values of the lead nucleus $I=0$ and $I=\frac{1}{2}$. The hyperfine structure due to the spin of the fluorine nucleus is neglected.

Vector $\boldsymbol{D}$ is the usual dipole moment of the molecule: $\boldsymbol{D}=D_{0} \boldsymbol{n}$, where $\boldsymbol{n}$ is the unit vector along the molecular axis. The magnetic moment $\boldsymbol{M}$ has components which are parallel and perpendicular to the vector $n$ :

$$
\begin{aligned}
& M_{0}=\left\langle\omega\left|\mu_{0}\right| \omega\right\rangle \\
& M_{1}=\left\langle\omega\left|\mu_{1}\right|-\omega\right\rangle \\
& \boldsymbol{\mu}=\boldsymbol{\mu}_{\mathrm{B}}(\boldsymbol{L}+2 \boldsymbol{S}) .
\end{aligned}
$$

Consider now a parity-non-conserving part of the spin-rotational Hamiltonian. The magnetic quadrupole moment at the lead is equal to zero and the corresponding term in the Hamiltonian vanishes. When the operator of the effective spin $s^{\prime}$ is used, the remaining terms are the same as for the ${ }^{2} \Sigma_{1 / 2}$ state. The latter were obtained by Flambaum and Khriplovich (1985b) and Kozlov (1985):

$$
H_{\mathrm{sr}}^{\prime}=\left(W_{\mathrm{P}, \mathrm{T}}^{\mathrm{d}} d_{\mathrm{e}}+W_{\mathrm{P}, \mathrm{T}}^{\mathrm{N}} x_{\mathrm{P}, \mathrm{T}}\right) s^{\prime} n+W_{\mathrm{P}} x_{\mathrm{P}} n \times s^{\prime} \cdot I
$$

where $d_{\mathrm{e}}$ is the electric dipole moment of the electron, $x_{\mathrm{P}, \mathrm{T}}$ is a constant of the P, T-odd neutral current, and $\chi_{\mathrm{P}}$ is a constant of the P-odd electron-nucleus interaction. This interaction is due to both the electron-nucleus neutral current and the anapole moment of the nucleus.

The values of these constants will be discussed in the last section of this paper.

The parameters $W$ are determined by the following expressions

$$
\begin{aligned}
& W_{\mathrm{P}, \mathrm{T}}^{\mathrm{d}}=\left(-8 \int_{0}^{\infty} g_{\mathrm{s}} \frac{\mathrm{d} U}{\mathrm{~d} r} g_{1} \mathrm{~d} r\right) a b \\
& W_{\mathrm{P}, \mathrm{T}}^{\mathrm{N}}=\left(2^{3 / 2} G \alpha Z \int_{0}^{\infty}\left(f_{\mathrm{s}} g_{1}+f_{1} g_{\mathrm{s}}\right) n(r) \mathrm{d} r\right) a b \\
& W_{\mathrm{P}}=\left(-2^{3 / 2} G \alpha \int_{0}^{\infty}\left(f_{\mathrm{s}} g_{1}+\frac{1}{3} f_{1} g_{\mathrm{s}}\right) n(r) \mathrm{d} r\right) a b .
\end{aligned}
$$


In these formulae $U$ is the electrostatic potential of the electron, $G$ is the Fermi constant and $n(r)$ is the nuclear density.

Results of the calculations of the spin-rotational Hamiltonian parameters are collected in table 3. The values of the rotational constant $B$ and $\omega$-doubling constant $\Delta$ are known from experiment (Huber and Herzberg 1979), $B=2288 \mathrm{~cm}^{-1}, \Delta=$ $-0.1388 \mathrm{~cm}^{-1}$.

In the above calculation two main simplifications were made:

(i) it is supposed that a simple wavefunction of the form (14) is sufficient for our purposes; and

(ii) the spin-orbit interaction for the valence electron is taken into account within the rough semiempirical model.

Table 3. Parameters of the spin-rotational Hamiltonian for the $\mathrm{X}^{2} \Pi_{1 / 2}$ state of $\mathrm{PbF}$.

\begin{tabular}{|c|c|c|c|c|c|c|c|}
\hline $\begin{array}{l}A_{\|} \\
(\mathrm{MHz})\end{array}$ & $\begin{array}{l}A_{-} \\
(\mathrm{MHz})\end{array}$ & $\begin{array}{l}D_{0} \\
\text { (D) }\end{array}$ & $\begin{array}{l}M_{0} \\
\left(\mu_{\mathrm{B}}\right)\end{array}$ & $\begin{array}{l}M_{1} \\
\left(\mu_{\mathrm{B}}\right)\end{array}$ & $\begin{array}{l}W_{\mathrm{P}, \mathrm{T}}^{\mathrm{d}} \\
\left(10^{19} \mathrm{MHz}\right. \\
\left.\mathrm{e}^{-1} \mathrm{~cm}^{-1}\right)\end{array}$ & $\begin{array}{l}W_{\mathrm{P}, \mathrm{T}}^{\mathrm{N}} \\
(\mathrm{KHz})\end{array}$ & $\begin{array}{l}W_{\mathrm{p}} \\
(\mathrm{KHz})\end{array}$ \\
\hline $9120+430^{a}$ & $-7850 \mp 390$ & 6.1 & $0.037 \pm 0.020$ & $0.25 \pm 0.06$ & $-1.4 \mp 0.4$ & $-75 \mp 24$ & $0.95 \pm 0.30$ \\
\hline
\end{tabular}

a The uncertainties in the table correspond to uncertainties in the molecular wavefunction (15).

It is known that wavefunctions similar to (14) are capable of reproducing the hyperfine structure of such molecules as $\mathrm{BaF}, \mathrm{HgF}$ and others (see, for example, Ernst et al 1986). The hyperfine interaction is as singular as the parity-non-conserving interactions. Thus we suppose that the wavefunction (14) is good enough to give the right values of the spin-rotational Hamiltonian parameters. Then the main errors in these parameters are due to the uncertainties in the coefficients (15).

\section{Conclusions}

Because of the large values of the $A$-tensor components, the spin-rotational spectrum of the ${ }^{207} \mathrm{PbF}$ molecule differs substantially from the pure rotational spectra of other isotopes. In particular, there are two levels of different parity separated by an interval which is approximately four times smaller than the splitting of the lowest $\omega$ doublet $\Delta$.

Unfortunately, these levels are not mixed by the operator (26), because they have different total angular momenta. The nearest levels which can be mixed by $H_{\mathrm{sr}}^{\prime}\left(J=\frac{3}{2}\right.$, $F=1, p= \pm 1$ ) are separated by an interval of the same order of magnitude as $\Delta$. Thus, the large hyperfine splitting in this molecule does not seem to bring any advantage to the experiment on $\mathrm{P}, \mathrm{T}$ non-conservation. However, a detailed investigation, based on the effective Hamiltonian (16), (25), (26) is desirable. Here we can only mention that the occupancies of the spin-rotational levels are inversely proportional to the factor $(2 I+1)$, and that the natural abundance of the spinless isotopes is about $77 \%$. Therefore, it seems more appropriate to use the spinless isotopes of lead for the search for $\mathrm{P}, \mathrm{T}$-odd effects in the PbF molecule. However only isotopes with non-zero spin may be used for the measurement of the constant $x_{\mathrm{P}}$.

Kozlov (1985) has examined the molecules with a ${ }^{2} \Sigma$ ground state; in particular, the molecule $\mathrm{HgF}$. The $W$ parameters for the $\mathrm{PbF}$ molecule appear to be several times smaller than those for $\mathrm{HgF}$. The main reason is the small value of the coefficient $a$ in 
the wavefunction (14). This coefficient enters the expressions (27)-(29). One can expect a similar relation for $W$ parameters of the heavy molecules in the ${ }^{2} \Sigma_{1 / 2}$ and ${ }^{2} \Pi_{1 / 2}$ ground states. Indeed, for the ${ }^{2} \Sigma_{1 / 2}$ states $a \simeq 1$, while for the ${ }^{2} \Pi_{1 / 2}$ states $a \simeq \eta \ll 1$ (see (8), (13) and (15)).

Finally, we shall briefly recall what is known about the constants of the Hamiltonian (26).

In the Salam-Weinberg theory the constant $x_{\mathrm{P}}$ of the electron-nucleon neutral current is determined by the Weinberg angle $\theta_{\mathrm{W}}$ :

$$
x_{\mathrm{P}}=1.25\left(1-4 \sin ^{2} \theta_{\mathrm{W}}\right)=0.1 \text {. }
$$

According to the estimate of Flambaum et al (1984), the contribution of the anapole moment of the lead nucleus to the constant $x_{\mathrm{P}}$ is of the same order of magnitude.

In the case of the P, T-odd interactions the theoretical situation is more uncertain. The present experimental bounds on the electron dipole moment and the constant $x_{\mathrm{P}, \mathrm{T}}$ are (Weiskopf et al 1968, Bouchiat 1975):

$$
\begin{aligned}
& d_{\mathrm{e}} \leqslant 3 \times 10^{-24} \text { e cm } \\
& x_{\mathrm{P}, \mathrm{T}} \leqslant 1 \times 10^{-3} .
\end{aligned}
$$

Now we can compare these bounds with our results. In the external electric field $E \simeq 3 \mathrm{kV} \mathrm{cm}^{-1}, D_{0} E \simeq B$, where $D_{0}$ and $B$ are the constants of the Hamiltonians (16) and (25). This means that the molecule is strongly polarised $\left(\left\langle\boldsymbol{s}^{\prime} \boldsymbol{n}\right\rangle= \pm \frac{1}{2}\right)$ and the energy shift due to the first two terms of the Hamiltonian (26) is close to its maximum value. When the direction of the electric field is reversed, the frequency shift of the magnetic resonance is

$$
\delta \nu=\delta \nu_{d}+\delta \nu_{x} \approx 2\left(W_{\mathrm{P}, \mathrm{T}}^{\mathrm{d}} d_{\mathrm{e}}+W_{\mathrm{P}, \mathrm{T}}^{\mathrm{N}} x_{\mathrm{P}, \mathrm{T}}\right) .
$$

The upper bounds on the $d_{\mathrm{e}}$ (31) and $x_{\mathrm{P}, \mathrm{T}}$ (32) correspond to $\left|\delta \nu_{x}\right| \simeq 150 \mathrm{~Hz}$, $\left|\delta \nu_{d}\right| \simeq 80 \mathrm{~Hz}$. The molecular beam resonance technique gives the opportunity of measuring frequency shifts a few orders smaller $\left(\sim 10^{-1}-10^{-2} \mathrm{~Hz}\right)$. Thus, the molecular beam experiment with the $\mathrm{PbF}$ molecule is rather promising.

\section{Acknowledgments}

The authors are grateful to I I Tupitsin for providing us with his results of the relativistic calculation of lead.

\section{References}

Almlöf J 1972 USIP report 72-09 University of Stockholm

Bagus P S 1975 IBM Res. Rep. RJ1077

Balasubramanian K $1985 \mathrm{~J}$. Chem. Phys. 832311

Bouchiat C 1975 Phys. Lett. B $\mathbf{5 7} 284$

Bratcev V F, Deyneka G E and Tupitsin I I 1977 Izv. Akad. Nauk SSR 412655

Coveney P V and Sandars P G H 1983 J. Phys. B: At. Mol. Phys. 163727

Danning T H and Hay P J 1977 Methods of Electronic Structure Theory vol 3, ed H F Schaefer (New York: Plenum) p 1

Dmitriev Yu Yu, Kozlov M G, Labzovsky L N and Titov A V 1985 Preprint LIYaF No 1046 (Leningrad)

Ernst W E, Kändler J and Törring T 1986 J. Chem. Phys. 844769 
Flambaum V V and Khriplovich I B 1985a Zh. Eksp. Teor. Fiz. 891505 1985b Phys. Lett. 110A 121

Flambaum V V, Khriplovich I B and Sushkov O P 1984 Phys. Lett. 146B 367

Gorshkov V G, Labzovsky L N and Moskalev A N 1979 Zh. Eksp. Teor. Fiz. 76414

Huber K P and Herzberg G 1979 Constants of Diatomic Molecules (New York: Van Nostrand)

Kahn L R 1984 Int. J. Quantum Chem. 25149

Khriplovich I B 1981 Nesokhranenie Chetnosty v Atomnikh Yavleniyakh (Parity Non-Conservation in Atomic Phenomena) (Moscow: Nauka)

Kozlov M G 1985 Zh. Eksp. Teor. Fiz. 891933

Kutzelnigg W 1984 Int. J. Quantum Chem. 25107

Liberman D, Waber J T and Cromer D T 1965 Phys. Rev. 137 A27

Sandars PG H 1967 Phys. Rev. Lett. 191396

Sobelman I 1963 Vvedenie v Teoriu Atomnikh Spektrov (Introduction to Atomic Spectra Theory) (Moscow: Nauka) $\$ 19$

Sushkov O P and Flambaum V V 1978 Zh. Eksp. Teor. Fiz. 751208

Sushkov O P, Flambaum V V and Khriplovich I B 1984 Zh. Eksp. Teor. Fiz. 871521

Vold T G, Raab E J, Heckel B and Fortson E N 1984 Phys. Lett. 522229

Wadt W R and Hay P J 1985 J. Chem. Phys. 82284

Weisskopf M C, Carrico I P, Gould H, Lipworth E and Stain T S 1968 Phys. Rev. Lett. 211645

Wilkening D A, Ramsey N F and Larson D J 1984 Phys. Rev. A 29425 